\title{
COVID-19 outcomes in patients with antiphospholipid syndrome: a retrospective cohort study
}

\author{
Erden $\mathrm{A}^{1}$, Karakas $\mathrm{O}^{1}$, Armagan $\mathrm{B}^{1}$, Guven $\mathrm{SC}^{1}$, Ozdemir $\mathrm{B}^{1}$, Atalar $\mathrm{E}^{1}$, Apaydin $\mathrm{H}^{1}$, Usul E , \\ Ates $\mathrm{I}^{3}$, Omma $\mathrm{A}^{1}$, Kucuksahin $\mathrm{O}^{4}$ \\ Ankara Sehir Hastanesi, MH6 Onkoloji binasi, Romatoloji bolumu, Bilkent, Cankaya, \\ Ankara, Turkey. drserdarguven@gmail.com
}

\begin{abstract}
BACKGROUND: Aim of this study is to investigate COVID-19 outcomes in patients with antiphospholipid syndrome (APS).

METHODS: A retrospective cohort was formed from APS patients. Patients were screened for a record of positive SARS-CoV 2 PCR. In PCR-positive patients, clinical data and information regarding COVID-19 outcomes were collected from medical records.

RESULTS: A positive PCR test was detected in 9/53 APS patients, while $66.7 \%, 33.3 \%$ and $11.1 \%$ of APS patients with COVID-19 were under hydroxychloroquine, LMWH or warfarin, and acetylsalicylic acid, respectively. There were $3 / 9$ patients found to be hospitalized and one died. No new thrombotic event was reported in any of the patients during COVID-19 infection.

CONCLUSION: Baseline use of hydroxychloroquine, antiaggregants and anticoagulants may be associated with an absence of new thrombotic event (Tab. 2, Ref. 33). Text in PDF www.elis.sk

KEY WORDS: antiphospholipid syndrome, COVID-19, outcome, thrombosis, mortality.
\end{abstract}

\section{Introduction}

Antiphospholipid syndrome (APS) is a systemic autoimmune disorder characterized by both venous and arterial recurrent thrombosis and/or obstetric morbidity with the presence of antiphospholipid (aPL) antibodies comprising lupus anticoagulant (LAC), anti- $\beta 2$-glycoprotein I (anti- $\beta 2 \mathrm{GPI}$ ) and anticardiolipin (aCL) (1).

Severe acute respiratory syndrome-coronavirus 2 (SARSCoV-2) infection, also known as coronavirus disease 2019 (COVID-19), can be asymptomatic but in severe cases it leads to pneumonia, acute respiratory failure, coagulopathy and even death $(2,3)$. There is a link between inflammation and the development of severe organ damage (4). Immunological studies have shown that the proinflammatory cytokines, interleukin (IL)-6, IL-17A and tumor necrosis factor (TNF) $\alpha$, are elevated in most patients with severe course (5). Hypercoagulation is an important conse-

${ }^{1}$ Ministry of Health Ankara City Hospital, Clinic of Rheumatology, Ankara, Turkey, ${ }^{2}$ Sincan Dr. Nafiz Korez State Hospital, Clinic of Emergency Medicine, Ankara, Turkey, ${ }^{3}$ University of Health Sciences, School of Medicine, Ministry of Health Ankara City Hospital, Department of Internal Medicine, Ankara, Turkey, and ${ }^{4}$ Ankara Yildirim Bayezit University, School of Medicine, Department of Internal Medicine, Division of Rheumatology, Ankara, Turkey

Address for correspondence: S.C. Guven, Ankara Şehir Hastanesi, MH6 Onkoloji binasi, Romatoloji bölümü, Bilkent, Çankaya, Ankara, Turkey, Postal code: 06800. Phone: 905337112951 quence of inflammation. Proinflammatory cytokines play a critical role in abnormal clot formation by platelet hyperactivation and inhibition of physiological anticoagulant pathways (6). COVID19-associated coagulopathy and diffuse intravascular coagulation have been associated with serious illness and death in COVID-19 (7). Various thrombotic events such as microvascular thrombosis, venous and pulmonary thromboembolism, and acute arterial thrombosis can be seen in critically ill patients without other risk factors for thrombosis (8).

So far, there is not enough data in the literature to enable us to evaluate the course of SARS-CoV-2 infection in patients with inflammatory rheumatic diseases. Yet, this group of patients seems to have similar results in terms of COVID-19 compared to those without rheumatic diseases $(9,10)$. There is scarcely any information in the literature regarding the course of COVID-19, especially in APS patients. In order to contribute to the literature on this issue, we aimed to evaluate the results of COVID-19 in our cohort of APS patients in terms of mortality, hospitalization and/ or intensive care unit (ICU) admission and length of hospital stay.

\section{Materials and methods}

This study was designed as a cross-sectional, retrospective cohort study with approval by Ankara City Hospital ethics committee and was therefore performed in accordance with the ethical standards laid down in the 1964 Declaration of Helsinki and its later amendments. An official permission was obtained from 
the Ministry of Health to conduct this study dated 20 February 2021.

A retrospective cohort was created among APS patients who met the Sapporo APS Classification Criteria (also called Sydney criteria (11)) and were previously followed by the authors (AE, OK AO, OK, SCG, BA) at Ankara City Hospital, Ankara Numune Training and Research Hospital and Ankara Atatürk Training and Research Hospital (the latter two centers were merged to found Ankara City Hospital in 2019 and have been working as a single center since clinical records of all three centers are accessible via Ankara City Hospital database). Between February 15 and February 22, 2021, the patients in this cohort were retrospectively investigated for the result of a nasopharyngeal swab for SARS-CoV-2 polymerized chain reaction (PCR) test from the Public Health Management System (HSYS). Patients older than 18 years of age with a recorded positive PCR test result from nasopharyngeal swab between July 1 and December 31, 2020 were enrolled in the study.

The length of hospital stay, rate of hospitalization, need for oxygen supplement, ICU admission and mortality due to COVID-19 were selected as main outcome variables and obtained from medical records and HSYS. Demographic data, comorbidities and medical treatment data of APS patients were collected from Ankara City Hospital database. In order to detect any thrombotic event developed in the post-COVID-19 period, the medical records of the patients up to three months after infection were scanned. Also, the patients whose information could not be obtained from the registry system were called by phone via numbers recorded in the hospital database.

The data were analyzed using Statistical Package for the Social Sciences (SPSS) 22.0 software. The normality of continuous variables was evaluated with Shapiro-Wilk test as well as visually with plots and histograms. Continuous variables were presented either with median (minimum-maximum) or mean \pm standard deviation, according to normality. Categorical variables are presented with numbers and percentages.

\section{Results}

Our cohort included a total of 53 APS patients. Among these, 9 patients were detected to have a positive SARS-CoV-2 PCR test during the pandemic. Demographics, comorbid diseases and drugs for APS treatment of these patients are given in Table 1.

Three of the 9 patients $(33.3 \%$ ) were found to be hospitalized for COVID-19 and one of them was admitted to ICU and died thereafter (Tab. 1). This patient was male, aged 68 years and had multiple comorbidities including coronary artery disease, hyper- tension and chronic kidney disease. He had thrombosis history thought to be related to APS. He was only under hydroxychloroquine (HCQ) (400 mg/day) treatment. Previously he was not taking any anticoagulant treatment. None of the other patients were admitted to ICU or died.

The median length of hospital stay was found to be 7.5 days. Among hospitalized patients, $66.7 \%$ had at least two comorbid diseases. An increasing trend in the frequency of comorbid diseases was observed in hospitalized patients (at least one comorbidity was present in $55.6 \%$ of all patients as compared to $66.7 \%$ in hospitalized patients). In our patient group, we did not observe any new thrombotic events during the treatment process or during the 3 -month post COVID-19 observation period. While 1 (16.7\%) of the patients who took HCQ needed hospitalization, 2 (66.7 \%) patients who did not take HCQ were hospitalized $(p=0.226)$.

Tab. 2. Distribution of aPL antibodies in antiphospholipid syndrome patients with COVID-19.

\begin{tabular}{lcc}
\hline & $\begin{array}{c}\text { All } \\
\text { (number }=9), \mathrm{n}(\%)\end{array}$ & $\begin{array}{c}\text { Hospitalized } \\
\text { (number }=3), \mathrm{n}(\%)\end{array}$ \\
\hline Lupus anticoagulant & $5(55.6)$ & $2(66.7)$ \\
Anticardiolipin Ig G & $1(11.1)$ & $0(0)$ \\
Beta 2 glicoprotein Ig M & $4(44.4)$ & $1(33.3)$ \\
Beta 2 glicoprotein Ig G & $2(22.2)$ & $1(33.3)$ \\
Beta 2 glicoprotein Ig A & $1(11.1)$ & $0(0)$ \\
Double positive & $1(11.1)$ & $0(0)$ \\
\hline COVID-19 - coronavirus disease 2019, aPLs - antiphospholipid antibodies, Ig - \\
immunoglobulin
\end{tabular}


120-124

Of the 9 APS and COVID-19 patients, five patients were LAC-positive and 4 patients were positive for aB2GP1 Ig M antibodies (Tab. 2). One had double positivity (aCL Ig G and aB2GP1 Ig $\mathrm{M}$ antibodies). Among the two patients who were hospitalized, one had LAC positivity alone and one had both aB2GP1 Ig M and Ig $\mathrm{G}$ positivity.

\section{Discussion}

In our study, $16.9 \%$ of the patients who were followed up for APS were diagnosed with COVID-19. The rate of hospitalization was $33.3 \%$, and the need for intensive care was $11.1 \%$. One patient $(11.1 \%)$ died which was an older patient compared to the others and had multiple comorbidities.

Some studies have shown that the use of antimalarials can alleviate the clinical symptoms of COVID-19 and delay disease progression without significant side effects $(9,10)$. In our study, only $1(11.1 \%)$ of the patients under HCQ treatment required hospitalization. In addition, none of the patients using antiaggregants/anticoagulants such as acetylsalicylic acid, warfarin or low molecular weight heparin (LMWH) required hospitalization.

In a study conducted in Hong Kong, 5 of 1,067 patients diagnosed with COVID-19 had rheumatological diseases (3 ankylosing spondylitis, 1 rheumatoid arthritis and 1 psoriatic arthritis) (12). It was determined that $0.12 \%$ (5 patients) of 39,835 rheumatological patients registered in Hong Kong developed COVID-19 when compared to $0.14 \%$ of the Hong Kong general population. Although there is no clear information in the literature regarding the incidence of COVID-19 in APS patients, in the study conducted in Hong Kong, the incidence of COVID-19 was found to be lower in those with rheumatological diseases than in the normal population (12). When the Global Rheumatology Alliance (GRA) COVID-19 database is examined, there are a total of 3,830 cases registered and their distribution is as follows: $37.4 \%$ $(1,394)$ rheumatoid arthritis, $11.8 \%(440)$ psoriatic arthritis, $11.6 \%$ (431) spondylitis, and $10.5 \%$ (391) systemic lupus erythematosus (13). There are 4,373 cases registered in the European League Against Rheumatism (EULAR) COVID-19 database until January 5, 2021, while $37 \%$ of them were followed with the diagnosis of rheumatoid arthritis, $15 \%$ with spondyloarthritis, $13 \%$ with psoriatic arthritis, $7 \%$ with systemic lupus erythematosus and $1 \%$ with Behçet's disease (14). When GRA data were examined, hospitalization rates were $46 \%$ for the patients with rheumatoid arthritis patients, $56 \%$ for systemic lupus erythematosus, and $30 \%$ for psoriatic arthritis (15). There was no further evaluation regarding APS in the GRA data. However, in our current study, the frequency of COVID-19 in APS patients was $16.9 \%$, the rate of hospitalization was $33.3 \%$ and mortality was seen in 1 patient. According to COVID-19 GRA physician-reported registry data, the mortality associated with COVID-19 among subjects with rheumatic disease was associated with commonly known factors (advanced age, male gender, certain comorbidities) and disease-specific factors (disease activity, specific medications) (13). In line with these studies, also in our study, the patient who died was an elderly ( 68 years old) male patient with multiple comorbidities and was not using any antiaggregants/anticoagulants for APS.

APS is characterized by arterial, venous or small-vessel thrombosis and/or recurrent pregnancy morbidities (16). The distinguishing feature of COVID-19 is hyperinflammation defined as "cytokine storm" driven by high levels of IL-1, IL-6, TNF $\alpha$ and other proinflammatory cytokines (17). Inflammation promotes thrombosis through various mechanisms, including activation of endothelial cells, platelets, monocytes, and tissue factor-factor VIIa pathway, and by altering fibrinolysis and natural anticoagulant pathways (thrombomodulin, protein $\mathrm{C}$ and $\mathrm{S}$, changes in tissue level etc.) (18). There are several features of the immunopathogenic pathway that associate COVID-19 and APS resulting in thrombosis (19). The three main factors responsible for activating immunoinflammatory responses and thrombosis appear to be the same in both diseases. These are upregulated cytokine secretion from innate immune system cells, thrombus formation and complement activation. The comorbidities that may worsen the course of COVID-19 are very similar to those suggested by the second hit hypothesis of APS (a second risk factor enhancing the thrombotic effects of APS (20)) which includes immunoinflammatory disorders such as metabolic syndrome, obesity, hypertension, and smoking (19). Additionally, COVID-19 and APS cases appear to respond well to anticoagulant therapy, and the beneficial effects observed with HCQ in APS patients have also been claimed for COVID-19 patients $(16,21)$.

The aPL antibodies target phospholipid proteins and their persistent presence is valuable in the diagnosis of APS. These antibodies can also occur temporarily in patients with critical illness and various infections. The aCL antibodies and LAC have been associated with a range of viral infections including hepatitis $\mathrm{C}$, acquired immunodeficiency syndrome, cytomegalovirus, varicella zoster, Epstein-Barr virus, adenovirus, and parvovirus B19 (22). The pathogenesis of COVID-19 is still under intense investigation, but growing evidence suggests that coagulopathy is a major factor in cases with worse outcomes $(23,24)$. The presence of aPL antibodies has been accepted as one of the mechanisms that cause hypercoagulation during COVID-19. In a case series of severe COVID-19 patients, high levels of aCL IgA antibodies and anti- $\beta 2$-GPI IgA as well as IgG antibodies were detected among 3 patients with multiple cerebral infarcts (25). Interestingly, IgA, the isotype that plays a particularly important role in mucosal immunity, has been identified as the most common aPL isotype detected in COVID-19 (26). Since COVID-19 mainly affects the pulmonary and intestinal mucosa, the increased production of the IgA isotype may be associated with impaired mucosal immune tolerance. IgA aß2GPI is significantly and independently associated with thrombosis in patients with systemic lupus erythematosus and APS (27). HCQ is an antimalarial drug that has been used for years in the treatment of autoimmune diseases due to its anti-inflammatory and immunomodulatory effects. Although the mechanism of action is unclear, it is also known to be beneficial in preventing thrombotic events while thrombotic events are less common in systemic lupus erythematosus patients receiving HCQ 
than in those who do not $(28,29)$. In studies conducted before the COVID-19 pandemic, it was reported that HCQ treatment was effective in preventing thrombotic events in patients with APS syndrome $(30,31)$. Nuri E et al. (32) reported that aPL titers and thrombotic events were lower in primary APS patients who received HCQ treatment than in those who did not. In the study of Sciascia et al (33), it was reported that pregnant women with aPL antibodies receiving HCQ treatment had a significantly higher rates of live births compared to those who did not receive HCQ treatment. In our study, we did not observe any new thrombotic events in the APS patient group during or after the COVID-19 process. Among our APS patients with COVID-19, $66.7 \%$ were under HCQ, $33.3 \%$ under low molecular weight heparin or warfarin, and $11.1 \%$ under acetyl salicylic acid. However, our sample size is not sufficient to evaluate as to whether these agents prevented thrombosis development during COVID-19 infection in APS patients.

Our study has several limitations. In addition to the natural limitations of a single-center retrospective case study, the number of evaluated patients is small and different results could be obtained by evaluating more patients. In addition, only 3 patients were hospitalized, and therefore, based on our results, the generalization regarding COVID-19 outcomes in APS patients may be disproportionate.

\section{Conclusion}

When compared to the results of other studies evaluating the course of COVID-19 in association with other rheumatologic diseases, our results of patients with APS have shown similar mortality and hospitalization rates. The presence of one or more comorbid diseases tended to be higher in hospitalized patients. HCQ use may be associated with decreased hospitalization rates in APS patients. However, due to the lack of literature data, larger studies are needed for more accurate results on COVID-19 outcomes in APS patients.

\section{References}

1. Miyakis S, Lockshin MD, Atsumi T et al. International consensus statement on an update of the classification criteria for definite antiphospholipid syndrome (APS). J Thromb Haemost 2006; 4 (2): 295-306.

2. Glass WG, Subbarao K, Murphy B, Murphy PM. Mechanisms of host defense following severe acute respiratory syndrome-coronavirus (SARSCoV) pulmonary infection of mice. J Immunol 2004; 173 (6): 4030-4039.

3. Tang N, Li D, Wang X, Sun Z. Abnormal coagulation parameters are associated with poor prognosis in patients with novel coronavirus pneumonia. J Thromb Haemost 2020; 18 (4): 844-847.

4. Miesbach W, Makris M. COVID-19: Coagulopathy, Risk of Thrombosis, and the Rationale for Anticoagulation. Clin Appl Thromb Hemost 2020; 26: 1076029620938149 .

5. Gralinski LE, Sheahan TP, Morrison TE et al. Complement Activation Contributes to Severe Acute Respiratory Syndrome Coronavirus Pathogenesis. mBio 2018; 9 (5): e01753-1178.
6. Dosquet C, Weill D, Wautier JL. Cytokines and thrombosis. J Cardiovasc Pharmacol 1995; 25 (Suppl 2): S13-19.

7. Zhou F, Yu T, Du R et al. Clinical course and risk factors for mortality of adult inpatients with COVID-19 in Wuhan, China: a retrospective cohort study. Lancet 2020; 395 (10229): 1054-1062.

8. Klok FA, Kruip MJHA, van der Meer NJM et al. Incidence of thrombotic complications in critically ill ICU patients with COVID-19. Thromb Res 2020; 191: 145-147.

9. Pablos JL, Abasolo L, Alvaro-Gracia JM et al. RIER investigators group. Prevalence of hospital PCR-confirmed COVID-19 cases in patients with chronic inflammatory and autoimmune rheumatic diseases. Ann Rheum Dis2020; 79 (9): 1170-1173.

10. Akiyama S, Hamdeh S, Micic D, Sakuraba A. Prevalence and clinical outcomes of COVID-19 in patients with autoimmune diseases: a systematic review and meta-analysis. Ann Rheum Dis 2020; annrheumdis-2020-218946.

11. Wilson WA, Gharavi AE, Koike T et al. International consensus statement on preliminary classification criteria for definite antiphospholipid syndrome: report of an international workshop. Arthritis Rheum 1999; 42 (7): 1309-1311.

12. So H, Mak JW, So J et al. Incidence and clinical course of COVID-19 in patients with rheumatologic diseases: A population-based study. Semin Arthritis Rheum 2020; 50 (5): 885-889.

13. Strangfeld A, Schäfer M, Gianfrancesco MA et al. COVID-19 Global Rheumatology Alliance. Factors associated with COVID-19-related death in people with rheumatic diseases: results from the COVID-19 Global Rheumatology Alliance physician-reported registry. Ann Rheum Dis 2021 annrheumdis-2020-219498.

14. Rheumatism, E.L.A., Eular COVID-19 database. 2021. https: //www. eular.org/myUploadData/files/eular_covid_19_registry_report_20210201. pdf

15. Gianfrancesco M, Hyrich KL, Al-Adely S et al. COVID-19 Global Rheumatology Alliance. Characteristics associated with hospitalisation for COVID-19 in people with rheumatic disease: data from the COVID-19 Global Rheumatology Alliance physician-reported registry. Ann Rheum Dis 2020; 79 (7): 859-866.

16. Rodziewicz M, D'Cruz DP. An update on the management of antiphospholipid syndrome. Ther Adv Musculoskelet Dis 2020; 12: $1759720 X 20910855$.

17. Huang C, Wang Y, Li X et al. Clinical features of patients infected with 2019 novel coronavirus in Wuhan, China. Lancet 2020; 395 (10223): 497-506.

18. Engelmann B, Massberg S. Thrombosis as an intravascular effector of innate immunity. Nature Rev Immunol 2013; 13 (1): 34-45.

19. Cavalli E, Bramanti A, Ciurleo R et al. Entangling COVID-19 associated thrombosis into a secondary antiphospholipid antibody syndrome: Diagnostic and therapeutic perspectives (Review). Int J Mol Med 2020; 46 (3): 903-912.

20. Nalli C, Andreoli L, Casu C, Tincani A. Management of recurrent thrombosis in antiphospholipid syndrome. Curr Rheumatol Rep 2014; 16 (3): 405.

21. McKee DL, Sternberg A, Stange U, Laufer S, Naujokat C. Candidate drugs against SARS-CoV-2 and COVID-19. Pharmacol Res 2020; 157: 104859 . 
120-124

22. Uthman IW, Gharavi AE. Viral infections and antiphospholipid antibodies. Semin Arthritis Rheum 2002; 31 (4): 256-263.

23. Li T, Lu H, Zhang W. Clinical observation and management of COVID-19 patients. Emerg Microbes Infect 2020; 9 (1): 687-690.

24. Han H, Yang L, Liu R et al. Prominent changes in blood coagulation of patients with SARS-CoV-2 infection. Clin Chem Lab Med 2020; 58 (7): 1116-1120.

25. Zhang Y, Xiao M, Zhang S et al. Coagulopathy and Antiphospholipid Antibodies in Patients with COVID-19. N Engl J Med 2020; 382 (17): e38.

26. Xiao M, Zhang $\mathbf{Y}$, Zhang $\mathbf{S}$ et al. Antiphospholipid Antibodies in Critically Ill Patients With COVID-19. Arthritis Rheumatol 2020; 72 (12): 1998-2004.

27. Andreoli L, Fredi M, Nalli C et al. Clinical significance of IgA anticardiolipin and IgA anti- $\beta 2$ glycoprotein I antibodies. Curr Rheumatol Rep 2013; 15 (7): 343.

28. Hunt BJ. A novel antiphospholipid antibody agent? Blood 2010; 115 (11): 2124-2125.
29. Wallace DJ, Gudsoorkar VS, Weisman MH, Venuturupalli SR. New insights into mechanisms of therapeutic effects of antimalarial agents in SLE. Nat Rev Rheumatol 2012; 8 (9): 522-533.

30. Schmidt-Tanguy A, Voswinkel J, Henrion D et al. Antithrombotic effects of hydroxychloroquine in primary antiphospholipid syndrome patients. J Thromb Haemost 2013; 11 (10): 1927-1929.

31. Mekinian A, Lazzaroni MG, Kuzenko A et al. SNFMI and the European Forum on Antiphospholipid Antibodies. The efficacy of hydroxychloroquine for obstetrical outcome in anti-phospholipid syndrome: Data from a European multicenter retrospective study. Autoimmun Rev 2015; 14 (6): 498-502.

32. Taraborelli M, Reggia R, Dall'Ara F et al. Longterm Outcome of Patients with Primary Antiphospholipid Syndrome: A Retrospective Multicenter Study. J Rheumatol 2017; 44 (8): 1165-1172.

33. Sciascia S, Hunt BJ, Talavera-Garcia E, Lliso G, Khamashta MA, Cuadrado MJ. The impact of hydroxychloroquine treatment on pregnancy outcome in women with antiphospholipid antibodies. Am J Obstet Gynecol 2016; 214 (2): 273.e1-273.e8. 